\title{
Effects of icariside II ameliorates diabetic cardiomyopathy in streptozotocin-induced diabetic rats by activating Akt/NOS/NF-кB signaling
}

\author{
LU YANG ${ }^{*}$, CHAOSHENG PENG ${ }^{*}$, JING XIA, WENLUO ZHANG, LI TIAN, \\ YUHONG TIAN, XIAOBIN YANG and YUEAN CAO
}

Special Care Medical Center, Navy General Hospital of PLA, Beijing 100048, P.R. China

Received January 22, 2017; Accepted October 10, 2017

DOI: $10.3892 / \mathrm{mmr} .2017 .8342$

\begin{abstract}
Icariside II is a flavonoid extracted from Epimedium that has antioxidant, anti-inflammatory and antiapoptotic effects. The aim of the present study was to evaluate the effects icariside II on diabetic cardiomyopathy in streptozotocin-induced diabetic rats. Icariside II treatment improved body weight, heart/body weight ratio and fasting blood glucose in diabetic model rats. Icariside II was demonstrated to reduce the expression levels of creatine kinase and lactate dehydrogenase in serum, and to lower cardiac oxidative stress, inflammation and apoptosis levels in diabetic rats. Icariside II treatment induced phosphoinositide 3-kinase and phosphorylated-Akt expression, and suppressed inducible nitric oxide synthase (iNOS) and nuclear factor $(\mathrm{NF})-\kappa \mathrm{B}$ protein expression in diabetic rat. Results from the present study suggested that treatment with icariside II improved diabetic cardiomyopathy in streptozotocin-induced diabetic rats by activating the $\mathrm{Akt} / \mathrm{NOS} / \mathrm{NF}-\kappa \mathrm{B}$ pathway.
\end{abstract}

\section{Introduction}

The incidence rate of diabetes, particularly type 1 , is rising rapidly worldwide and increases by $>7$ million every year (1). In China, the diabetes incidence rate has become increasingly urgent and severe; the New England Journal of Medicine reported that China topped the list in the number of diabetic patients in the world in 2010 (2). Currently, 1 patient succumbs to diabetes every $10 \mathrm{sec}$ on average around the world, and the average annual cost for

Correspondence to: Professor Yuean Cao, Special Care Medical Center, Navy General Hospital of PLA, 6 Fucheng Road, Beijing 100048, P.R. China

E-mail: caoyuean@163.com

${ }^{*}$ Contributed equally

Key words: icariside II, diabetic cardiomyopathy, diabetic, Akt, inducible nitric oxide synthase, nuclear factor- $\kappa \mathrm{B}$ the treatment of diabetes is $>\$ 200$ billion, accounting for $\sim 18.2 \%$ of total medical expenses $(3,4)$. Diabetes severely affects human health, and patients with diabetes often experience complications in heart, brain and kidney, among which cardiovascular diseases are the main cause of mortality in patients with diabetes (5).

With rapid economic development, prolonging lifespans and changes in lifestyle, cardiovascular diseases have become the major cause of mortality in China (6). As a major risk factor of cardiovascular disease, diabetes increases the incidence of cardiovascular diseases (2). Previous studies have reported that mortality from cardiovascular complications in patients with diabetes is 3-5 times higher compared with those without diabetes, and the incidence rate of ischemic heart disease in patients with diabetes is twice that of those without diabetes $(6,7)$.

The phosphoinositide 3-kinase (PI3K)/Akt signaling pathway participates in cell differentiation, proliferation, apoptosis and migration, and overactivation of this pathway may lead to cellular dysfunction (8). PI3K/Akt signaling pathway serves biological functions through activation of a number of processes (9). It has been $>10$ years since the PI3K/Akt pathway was first identified; it was initially examined in studies on the metabolism, differentiation, proliferation, apoptosis and angiogenesis of tumor cells (9). The biological functions of PI3K/Akt signaling occur through the activation and phosphorylation of Akt (10). The PI3K/Akt/endothelial nitric oxide synthase (eNOS)/nitric oxide (NO) pathway is a cellular 'survival signal' pathway that serves an important role in protecting the cardiovascular system (11).

As a nuclear transcription factor with wide biological activities, nuclear factor (NF)- $\mathrm{B}$ serves an important role in signal transduction and gene expression (11). High blood glucose levels may improve the activity of NF- $\mathrm{NB}$, and activated $N F-\kappa B$ is involved in the production of a number of inflammatory cytokines, proliferation and differentiation of fibroblasts, as well as extracellular matrix cross-linking and cellular apoptosis (12). NF- $\kappa \mathrm{B}$ also regulates the transcriptional activity of NOS and cyclooxygenase-2, along with other DNA binding proteins (13).

Icariside II is a flavonoid extracted from Epimedium that has been reported to reduce the expression of rat hippocampal 
brain-derived neurotrophic factor, induced by D-galactose and its receptor TrkB tyrosine kinase, to facilitate axon regeneration, thus promoting nerve regeneration in rats with dementia and improving their cognitive function (14). Previous studies have also demonstrated that Epimedium, icariside II and Epimedium extract aid in recovering the functions of injured peripheral nerves $(14,15)$. The present study aimed to investigate whether icariside II treatment was able to protect against diabetic cardiomyopathy in streptozotocin-induced type I diabetic rats and to explore the underlying molecular mechanism in vivo.

\section{Materials and methods}

Animal model and treatment. Male Sprague-Dawley rats (weight, 180-200 g; age 7-8 weeks) were purchased from the Experimental Animal Center of Beijing University (Beijing, China), housed at $22-23^{\circ} \mathrm{C}, 55-60 \%$ humidity, SPF atmosphere $\left(0.03 \% \mathrm{CO}_{2}\right), 12 \mathrm{~h}$ light/dark cycle and freely access to food and water. All experiments in this study were approved by the Institutional Animal Care and Use Committee of Navy General Hospital of PLA (Beijing, China). A total of 36 rats were randomly divided into three groups ( $n=12 /$ group): i) Normal control group (Control group) group, which received an intraperitoneal (i.p.) injection of citrate-phosphate buffer (0.1 mol/l); ii) streptozotocin (STZ)-induced diabetic rat group (STZ group), which received an i.p. injection of STZ $(60 \mathrm{mg} / \mathrm{kg}$; Sigma-Aldrich; Merck KGaA, Darmstadt, Germany) to induce type 1 diabetes; and iii) STZ-induced diabetic rats + icariside II therapy group (STZ + icariside II group), which received an i.p. injection of STZ $(60 \mathrm{mg} / \mathrm{kg})$, followed by icariside II (5 mg/kg/day; Sigma-Aldrich; Merck KGaA) i.p. injection for 8 weeks. Following 8 weeks of treatment, body weight and heart weight were measured and calculated as (heart weight)/(body weight).

Measurement of fasting blood glucose. Following treatments, blood was collected by tail vein puncture and the fasting blood glucose levels (following $12 \mathrm{~h}$ of fasting) under anesthesia (35 mg/kg pentobarbital sodium) were measured using a glucometer (Sinocare Inc., Changsha, China). The serum was separated by centrifuging at $1,500 \times \mathrm{g}$ for $10 \mathrm{~min}$ at $4^{\circ} \mathrm{C}$ and stored at $-70^{\circ} \mathrm{C}$ until use.

Inflammatory cytokines detection and oxidative stress measurement. Serum creatine kinase (CK, cat. no. A032) and lactate dehydrogenase ( $\mathrm{LDH}$, cat. no. A020-2) levels were measured using ELISA kits (Nanjing Jiancheng Biology Eng ineering Institute). Protein homogenates were prepared from cardiac tissues in Lysis Buffer (Nanjing Keygen Biotech Co., Ltd., Nanjing, China) on ice for $15 \mathrm{~min}$, and protein contents were determined using the bicinchoninic acid protein assay kit (Nanjing Keygen Biotech Co., Ltd.). Tumor necrosis factor- $\alpha$ (TNF- $\alpha$, cat. no. H052), interleukin (IL)-1 $\beta$ (cat no. H002) and interleukin-6 (IL-6, cat. no. H007), malondialdehyde (MDA, cat. no. A003-1), superoxide dismutase (SOD, cat. no. A001-1), glutathione (GSH, cat. no. A006-2) and GSH peroxidase (GPx, cat. no. A005) expression levels were measured using ELISA kits (Nanjing Jiancheng Bioengineering Institute, Nanjing, China). The absorbance was determined using an automatic microplate reader (PerkinElmer, Inc., Waltham, MA, USA) at $450 \mathrm{~nm}$.

Western blotting. Protein homogenates were prepared from cardiac tissues in Lysis Buffer (Nanjing Keygen Biotech Co., Ltd., Nanjing, China) on ice for $15 \mathrm{~min}$, and protein contents were determined using the BCA Protein Assay kit (Nanjing Keygen Biotech Co., Ltd.). Equal amounts of protein $(50 \mu \mathrm{g})$ were separated by $8-12 \%$ SDS/PAGE and transferred to nitrocellulose membranes (EMD Millipore, Billerica, USA). Following blocking with 5\% nonfat milk for $1 \mathrm{~h}$, the membranes were individually incubated with primary antibodies (Santa Cruz Biotechnology, Inc., Dallas, TX, USA) against apoptosis regulator $\mathrm{Bax}$ (cat. no. sc-526, 1:500), caspase-3 (cat. no. sc-98785, 1:500), PI3K (cat. no. sc-7174, 1:500), phosphorylated (p)-Akt (cat. no. sc-135650, 1:500), inducible (i) NOS ( cat. no. sc-649, 1:500), NF-кB (cat. no. sc-109, 1:500) and GAPDH (cat. no. sc-25778, 1:500, to normalize protein expressions) overnight at $4{ }^{\circ} \mathrm{C}$ with agitation. Membranes were washed with TBS with $0.1 \%$ Tween-20, incubated with horseradish peroxidase-conjugated secondary antibody (cat. no. sc-2030, 1:2,000; Santa Cruz Biotechnology, Inc.) at room temperature for $1 \mathrm{~h}$ and were visualized using an Enhanced Chemiluminescence Detection kit (GE Healthcare, Chicago, IL, USA and analyzed Image-Pro Plus 6.0 software (Media Cybernetics, Inc., Rockville, MD,USA).

Statistical analysis. All data are expressed as the mean \pm standard deviation. Statistical significance was analyzed by one-way analysis of variance and Tukey's post hoc test using SPSS version 18.0 (SPSS, Inc., Chicago, IL, USA). P<0.05 was considered to indicate a statistically significant difference.

\section{Results}

Icariside II treatment improves body weight, heart/body weight Ratio and fasting blood glucose in diabetic rat. Body weight was significantly reduced in rats in the STZ group compared with Control rats (Fig. 1A). Treatment with STZ resulted in significant increases in the heart/body weight ratio and fasting blood glucose compared with the untreated Control group (Fig. 1B and C, respectively). Notably, rats co-treated with STZ and icariside II exhibited a significant increase in body weight and significant reductions in heart/body weight ratios and fasting blood glucose levels, compared with diabetic rats in the STZ group (Fig. 1).

Icariside II lowers $C K$ and LDH levels in the serum of diabetic rats. To investigate whether icariside II protected against diabetic cardiomyopathy, serum $\mathrm{CK}$ and $\mathrm{LDH}$ levels were measured. $\mathrm{CK}$ and $\mathrm{LDH}$ levels in the serum of diabetic rats were higher compared with the Control group (Fig. 2A and B, respectively). Co-treatment with icariside II significantly reduced $\mathrm{CK}$ and $\mathrm{LDH}$ levels in serum of diabetic rat (Fig. 2).

Icariside II reduces cardiac inflammation in diabetic rats. To detect whether icariside II treatment in diabetic rats exhibited anti-inflammatory effects, the expression levels of TNF- $\alpha$, IL- $1 \beta$ and IL- 6 in myocardial tissues were measured by ELISA. TNF- $\alpha$, IL-1 $\beta$ and IL- 6 levels in heart tissue were 
A

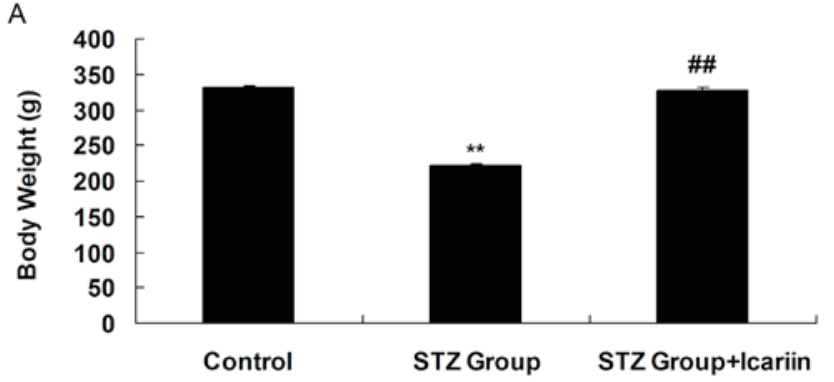

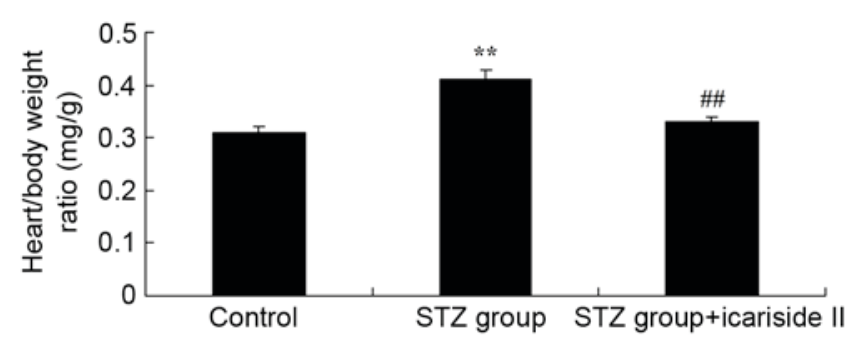

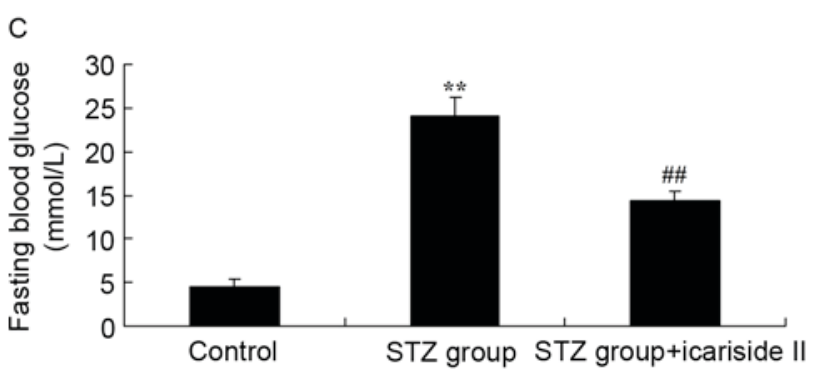

Figure 1. Icariside II treatment improves body weight, heart/body weight ratio and fasting blood glucose levels in diabetic rats. Icariside II treatment improved (A) body weight, (B) heart/body weight ratio and (C) fasting blood glucose in diabetic rats. ${ }^{* *} \mathrm{P}<0.01$ vs. Control group; ${ }^{\# \#} \mathrm{P}<0.01$ vs. STZ group. STZ, streptozotocin.
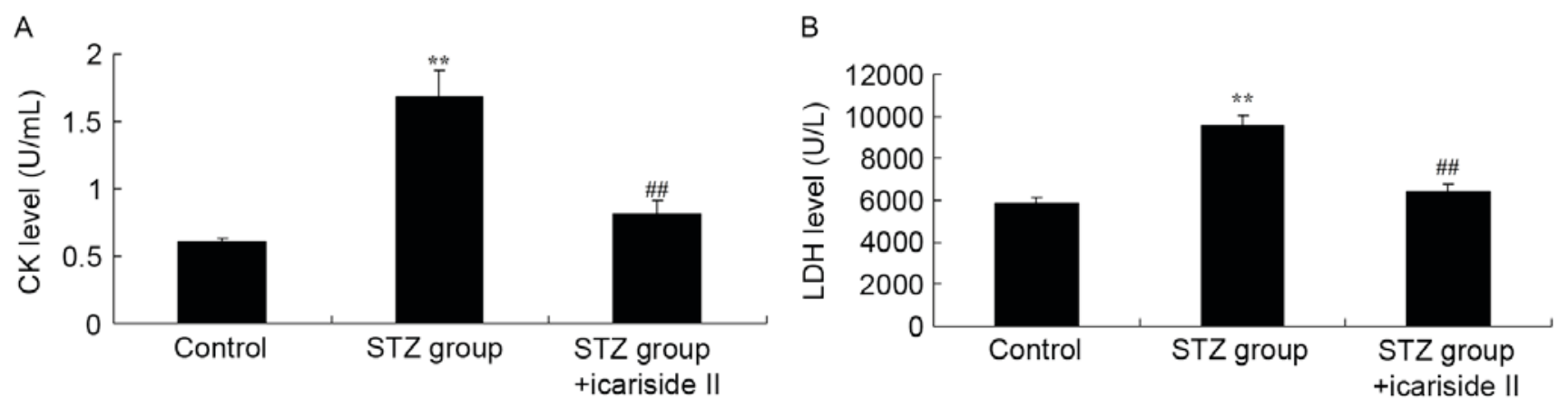

Figure 2. Icariside II treatment lowers CK and LDH levels in the serum of diabetic rats. Icariside II treatment lowers (A) CK and (B) LDH levels in the serum of diabetic rats. ${ }^{* *} \mathrm{P}<0.01$ vs. Control group; ${ }^{\# \#} \mathrm{P}<0.01$ vs. STZ Group. CK, creatine kinase; LDH, lactate dehydrogenase; STZ, streptozotocin.
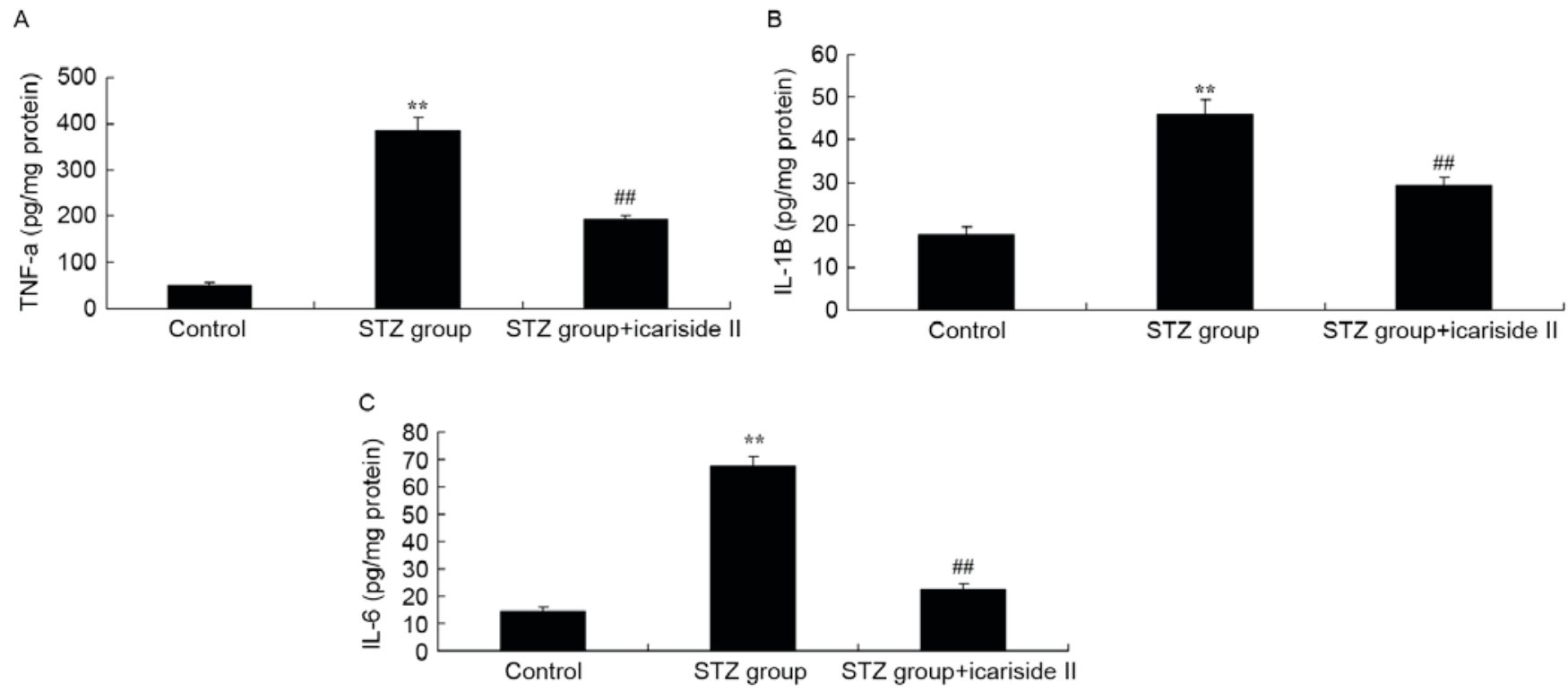

Figure 3. Icariside II reduces cardiac inflammation in diabetic rats. Icariside II co-treatment reduced the expression levels of (A) TNF- $\alpha$, (B) IL-1 $\beta$ and (C) IL-6 in myocardial tissues. ${ }^{* *} \mathrm{P}<0.01$ vs. Control group; ${ }^{\# \#} \mathrm{P}<0.01$ vs. STZ Group. IL, interleukin; STZ, streptozotocin; TNF, tumor necrosis factor. 
A

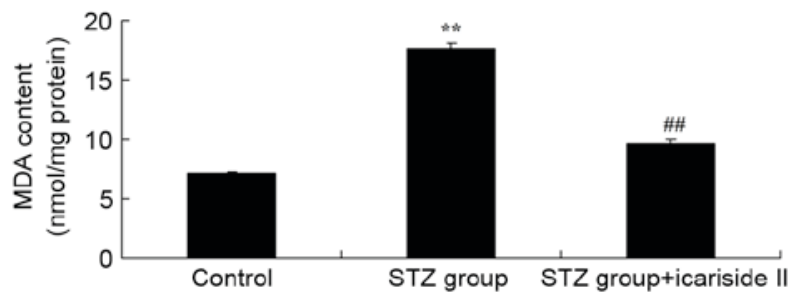

C

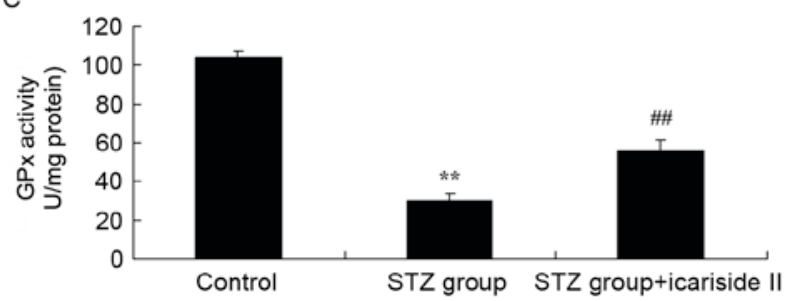

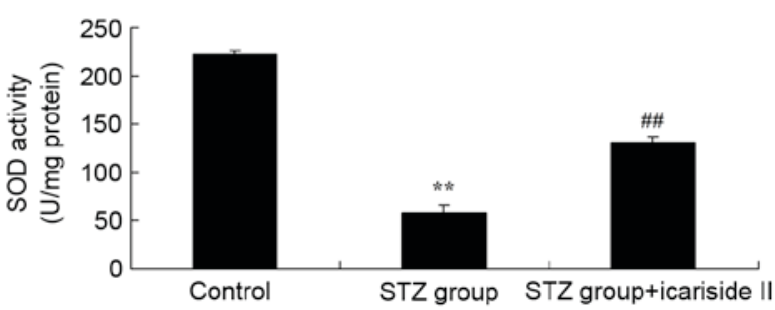

D

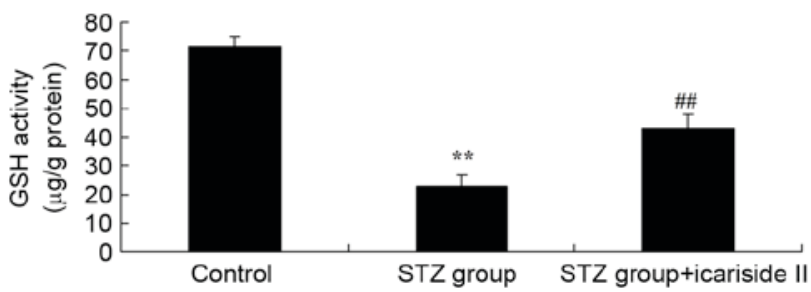

Figure 4. Icariside II reduces cardiac oxidative stress in diabetic rats. Icariside II co-treatment reduces cardiac expression levels of (A) MDA, (B) SOD, (C) GPx and (D) GSH. ${ }^{* *} \mathrm{P}<0.01$ vs. Control group; ${ }^{\# \#} \mathrm{P}<0.01$ vs. STZ group. GPx, glutathione peroxidase; GSH, glutathione; MDA, malondialdehyde; SOD, superoxide dismutase; STZ, streptozotocin.
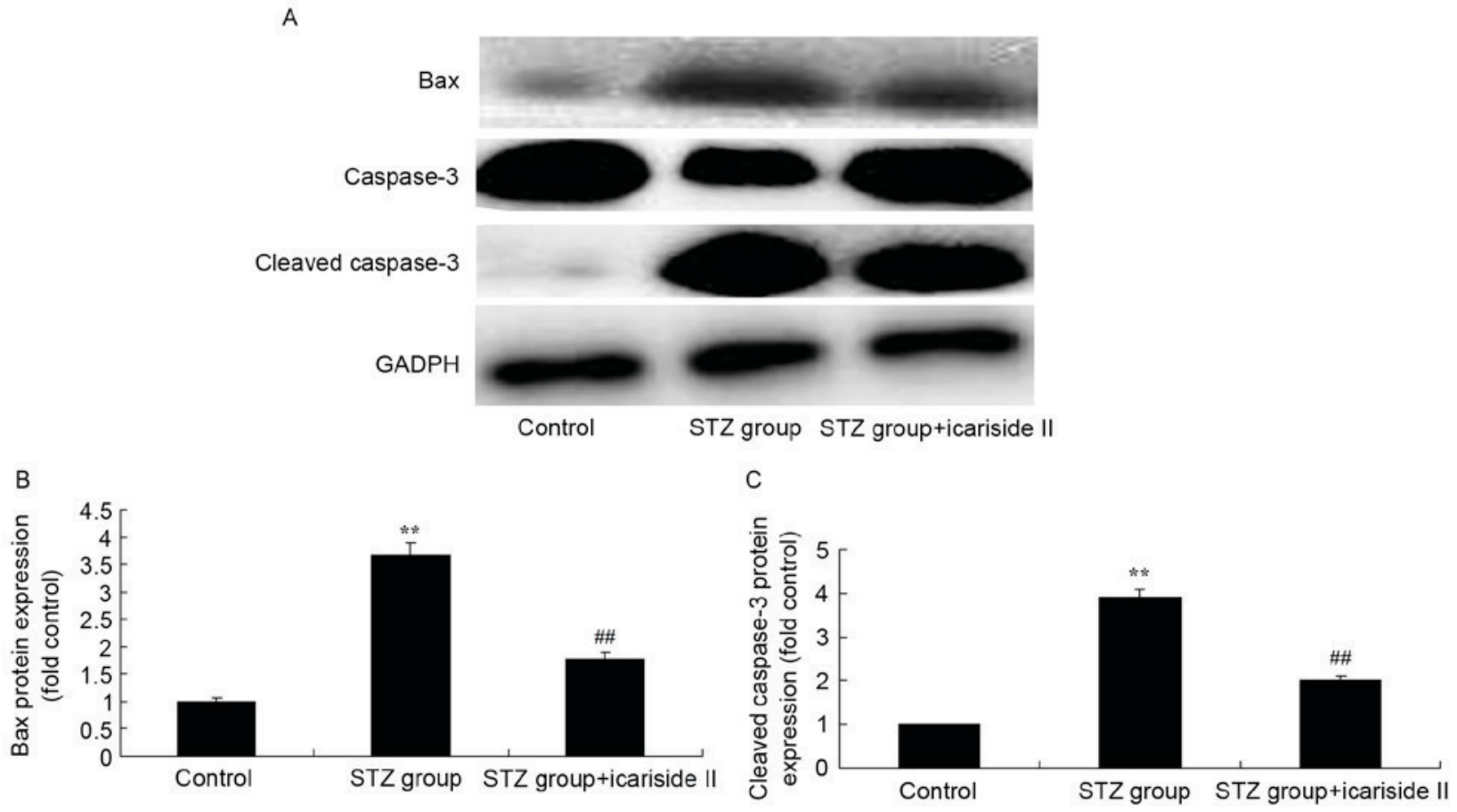

Figure 5. Icariside II decreases cardiac apoptosis levels in diabetic rats. (A) Icariside II co-treatment decreases cardiac expression levels of Bax and caspase-3 protein as detected by western blotting assays. Densitometric analysis of (B) Bax and (C) caspase-3 protein expression from the western blotting in part (A). ${ }^{* *} \mathrm{P}<0.01$ vs. Control group; ${ }^{\# \#} \mathrm{P}<0.01$ vs. STZ group. STZ, streptozotocin.

significantly increased in STZ-treated diabetic rats (Fig. 3A-C, respectively). Rats co-treated with icariside II exhibited reduced levels of TNF- $\alpha$, IL-1 $\beta$ and IL- 6 compared with the STZ-only group (Fig. 3).

Icariside II reduces cardiac oxidative stress in diabetic rats. To evaluate the anti-oxidative effects of icariside II treatment in diabetic rats, the expression levels of MDA, SOD, GPx and GSH in myocardial tissues were assessed by ELISA. Rats treated with STZ exhibited a significant increase in MDA content (Fig. 4A), and a reduction in SOD, GPx and GSH levels compared with the Control rats (Fig. 4B-D, respectively). STZ-induced diabetic rats co-treated with icariside II exhibited a decrease in MDA and an increase in SOD, GPx and GSH levels (Fig. 4). These results indicated that icariside II treatment may attenuate diabetes-induced oxidative stress in heart tissues.

Icariside II treatment decreases cardiac apoptosis levels in diabetic rats. To further investigate the potential anti-apoptotic role of icariside II in diabetic rats, the levels of Bax and caspase-3 protein expression were measured by western blotting (Fig. 5A). Bax and cleaved caspase- 3 protein expression in diabetic rats in 
A
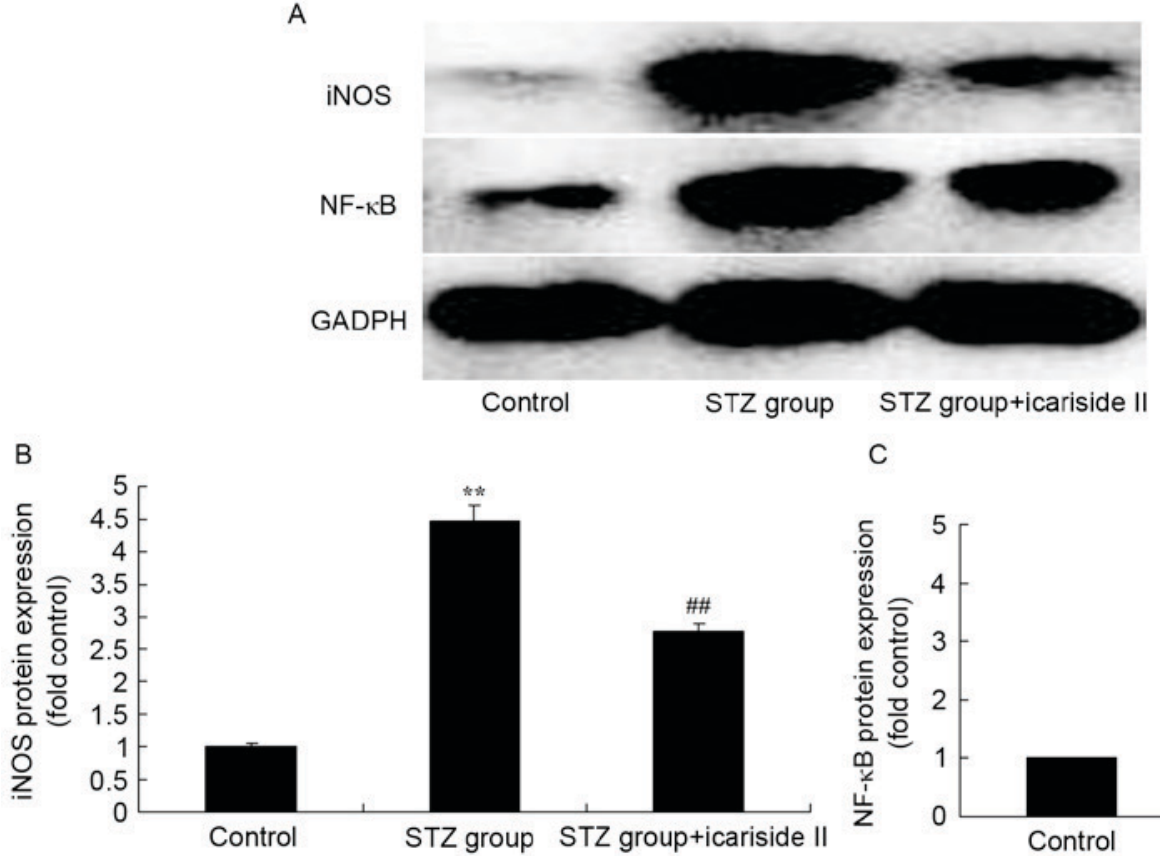

C

Figure 6. Icariside II reduces iNOS and NF- $\mathrm{kB}$ protein expression in diabetic rats. (A) Icariside II co-treatment reduced iNOS and NF- $\mathrm{B}$ protein expression as demonstrated by western blotting assays. Densitometric analysis of (B) iNOS and (C) NF-kB protein expression from the western blotting in part (A). ${ }^{* *} \mathrm{P}<0.01$ vs. Control group; ${ }^{\#} \mathrm{P}<0.01$ vs. STZ group. iNOS, inducible nitric oxide synthase; NF, nuclear factor; STZ, streptozotocin.
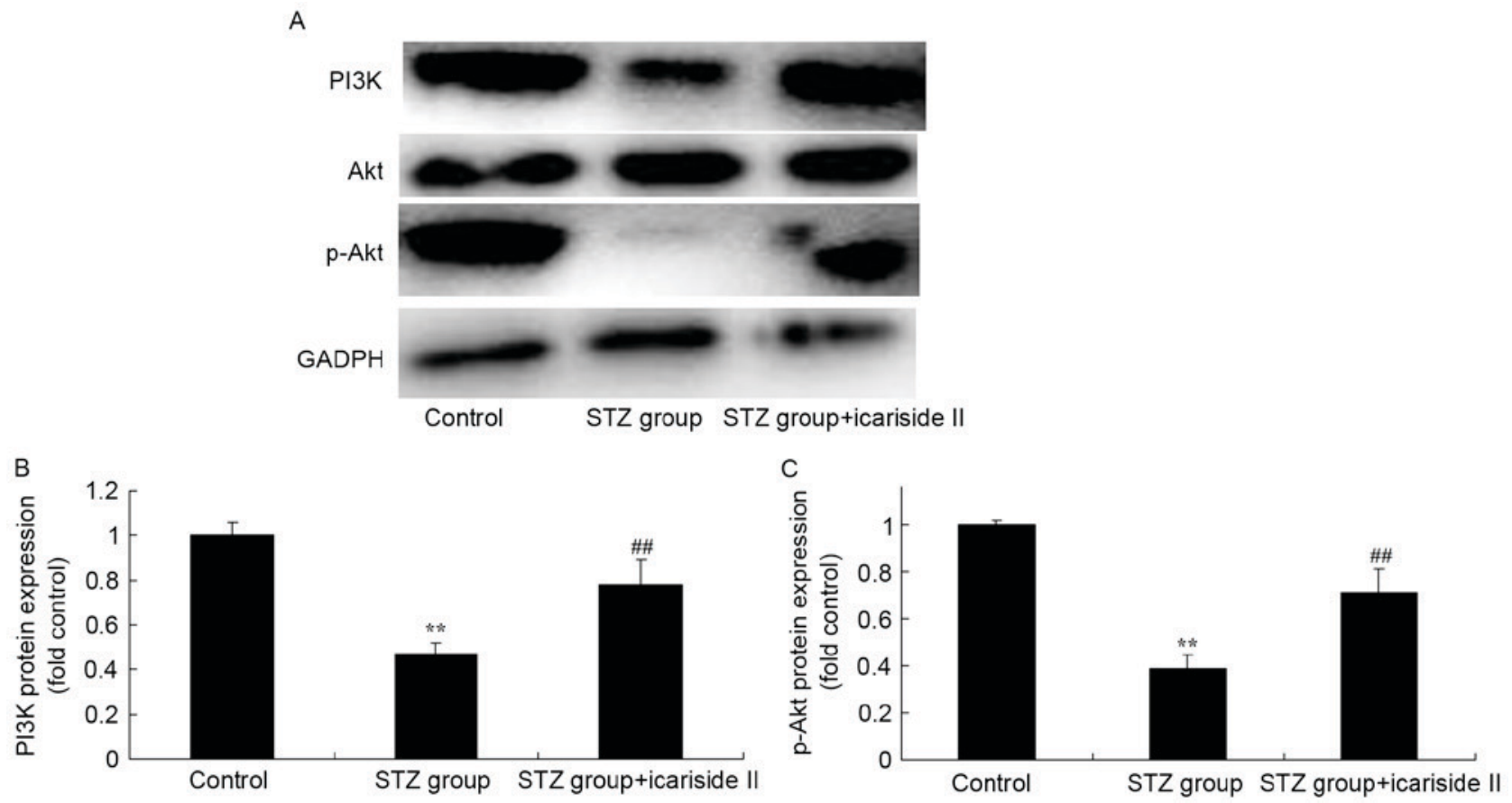

Figure 7. Icariside II induces PI3K and p-Akt protein expression in diabetic rats. (A) Icariside II induced PI3K and p-Akt protein expression, as detected by western blotting assays. Densitometric analysis of (B) PI3K and (C) p-Akt protein expression levels from the western blotting in part (A).** $\mathrm{P}<0.01 \mathrm{vs.} \mathrm{Control}$ group; ${ }^{\#} \mathrm{P}<0.01$ vs. STZ group. P, phosphorylated; PI3K, phosphoinositide 3-kinase; STZ, streptozotocin.

STZ group were significantly higher compared with expression levels in the Control group (Fig. 5B and C, respectively). Icariside II co-treatment significantly reduced Bax and cleaved caspase-3 protein expression in the heart tissues of diabetic rats (Fig. 5).

Icariside II reduces iNOS and $N F-\kappa B$ protein expression in diabetic rats. To further elucidate the potential regulatory mechanism of icariside II on iNOS and NF- $\kappa$ B protein expression in STZ-induced diabetic rats, iNOS and $\mathrm{NF}-\kappa \mathrm{B}$ protein expression levels were measured using in cardiac tissue by western blotting (Fig. 6A). Results from western blotting demonstrated that iNOS and NF- $\mathrm{NB}$ protein expression levels of diabetic rats were significantly higher compared with rats in the Control group (Fig. 6B and C, respectively). Icariside II 
co-treatment significantly reduced iNOS and NF- $\kappa \mathrm{B}$ protein expression in STZ-induced diabetic rats (Fig. 6).

Icariside II induces PI3K and $p$-Akt protein expression in diabetic rats. To further assess whether the PI3K/Akt signaling pathway is a functional target of icariside II, the PI3K/Akt signaling pathway was examined in cardiac tissue using western blotting (Fig. 7A). Significant reductions in PI3K and $\mathrm{p}$-Akt protein expression in heart tissues of diabetic rats were observed, compared with the Control group (Fig. 7B and $\mathrm{C}$, respectively). Icariside II co-treatment significantly induced PI3K/Akt signaling in heart tissues of diabetic rats (Fig. 7).

\section{Discussion}

Diabetic cardiomyopathy is a severe diabetic complication that is characterized by ventricular diastolic or systolic dysfunction (16). As the number of people diagnosed as obese increases, the number of patients with diabetes continue to rise, and diabetic cardiomyopathy has become an urgent clinical problem that needs to be solved (17). A previous study reported that diabetes increases the risk of heart failure by 2-3 times, and even accelerates the deterioration of heart function in patients with hypertension, ischemic heart disease and atrial fibrillation (18). Inflammatory reactions and apoptosis in myocardial tissues are key mechanisms in the incidence and development of diabetic cardiomyopathy. The present study demonstrated that icariside II treatment improved body weight, heart/body weight ratio and fasting blood glucose, and reduced CK and LDH levels in the serum of STZ-induced type I diabetic rats. A previous study demonstrated that icariside II reduced the STZ-induced cognitive deficits in rats through anti-inflammatory effects (14).

Oxidative stress has also been demonstrated to be an important factor that may lead to the occurrence and development of type 2 diabetes (19). Oxidative stress is an imbalance between the production and removal of reactive oxygen species (ROS), leading to excessive production of ROS and reactive nitrogen species, thereby injuring tissue cells and biological macromolecules, including proteins and nucleic acids (20). However, obesity or high glucose levels may significantly increase the production of superoxide, and oxidative stress is induced when the production rate of superoxide exceeds its removal rate (21). A previous study has demonstrated that oxidative stress may lead to functional injury of islet $\beta$ cells and peripheral insulin resistance, thereby inducing diabetes and even diabetic neuropathy, diabetic retinopathy and diabetic cardiovascular disease and other complications under the severe cases (22). In the present study, icariside II treatment was demonstrated to reduce the expression levels of TNF- $\alpha$, IL-1 $\beta$ and IL- 6 levels in the heart tissue of STZ-induced type I diabetic rats. A previous study revealed that icariside II reduces diabetic nephropathy in STZ-induced diabetic rats through antioxidative effects (15).

Apoptosis serves an important role in diabetic myocardial injury and is one of the important manifestations of ischemic myocardial injury (23). Experimental results have shown that ischemia/reperfusion-induced apoptotic injuries in rats with diabetes are more serious than in rats without diabetes. Previous studies also have demonstrated that myocardial cell injury due to apoptosis may be an important cause of various heart diseases $(23,24)$. As myocardial cell apoptosis is the main pathological mechanism of ischemia/reperfusion injury, the development of antiapoptotic drugs is required to prevent further injury to cardiomyocytes and to reduce ischemia/reperfusion-induced myocardial injury, thus slowing down or preventing the occurrence of various heart diseases, which may provide a new strategy for the treatment of cardiovascular complications in patients with diabetes (25). The present study demonstrated that icariside II treatment significantly suppressed the protein expression levels of Bax and cleaved caspase- 3 in heart tissue of type I diabetic rats.

Activation of PI3K-Akt signaling may reduce apoptosis and regulate glucose transport and glycogen synthesis (26). Akt may have a cytoprotective function by activating its downstream effector molecules, such as eNOS and anti-apoptotic Bcl-2, to prevent cell death through the mitochondrial pathway (27). The present data revealed that icariside II significantly suppressed iNOS and $\mathrm{NF}-\kappa \mathrm{B}$ protein expression in the heart tissue of type I diabetic rats. A previous study reported that icariside II protects dexamethasone treated osteoblasts and activates epidermal growth factor receptor/Akt/nuclear factor erythroid 2-related factor 2 signaling (28).

As a type of information transfer molecule, NO easily diffuses with only several sec of half-life, which makes it difficult to directly determine NO levels in tissues (29). iNOS expression is regulated by a number of transcription factors, including $N F-\kappa B(29)$. NF- $\kappa \mathrm{B}$ is activated at the initial stage of inflammation to upregulate the expression of iNOS and to increase the production of NO (20). In addition, iNOS and proinflammatory factors improve the activity of $\mathrm{NF}-\kappa \mathrm{B}$, leading to the expression of $\mathrm{NF}-\kappa \mathrm{B}$ inflammatory protein target genes in large amount, thus promoting the development of inflammation (13). Results from the present study suggested that icariside II significantly induced PI3K/Akt signaling in heart tissue of type I diabetic rats. A previous study demonstrated that icariside II inhibited the epithelial-mesenchymal transition of A549 and H1299 lung carcinoma cells in an inflammatory microenvironment, through the Akt/NF- $\kappa \mathrm{B}$ signaling pathway (30). Another study reported that icariside II induced apoptosis in human PC-3 prostate cancer cells through iNOS expression (31).

In conclusion, the present study demonstrated that treatment with icariside II reduced diabetic cardiomyopathy in STZ-induced diabetic rats through anti-inflammatory, anti-oxidative stress and antiapoptotic effects. Future studies will investigate the morphological alterations in cardiac tissues and assess cardiac function in response to icariside II treatment in a mouse model of diabetes. In addition, future studies will aim to explore the effects of icariside II on glucose metabolism and cardiomyopathy in a mouse model of type II diabetes. It is thus suggested that icariside II may be a promising candidate drug for the clinical treatment of diabetic cardiomyopathy through the Akt/NOS/NF- $\kappa \mathrm{B}$ pathway.

\section{Acknowledgements}

The present study was supported by grants from the the National Natural Science Foundation of China (grant no. 81671279), the Natural Science Foundation of Hainan 
Province (grant no. 20168361), the Science and Technology Special Foundation for Social Development in Hainan Province (grant no. 2015SF05) and Sanya Municipal Scientific and Technological Innovation Project in Health Care (grant no. 2014YW41).

\section{References}

1. Agrawal V, Agrawal A, Dwivedi AN and Tripathi K: Correlation between $2 \mathrm{D}$ echocardiography and multidetector row $\mathrm{CT}$ for early detection of diastolic dysfunction in normotensive diabetic patients. J Clin Diagn Res 10: OC27-30, 2016.

2. Ding Y, Wang Y, Chen J, Hu Y, Cao Z, Ren P and Zhang Y: p21 overexpression sensitizes osteosarcoma U2OS cells to cisplatin via evoking caspase-3 and $\mathrm{Bax} / \mathrm{Bcl}-2$ cascade. Tumour Biol 35: 3119-3123, 2014

3. Pham I, Cosson E, Nguyen MT, Banu I, Genevois I, Poignard P and Valensi P: Evidence for a specific diabetic cardiomyopathy: An observational retrospective echocardiographic study in 656 asymptomatic type 2 diabetic patients. Int J Endocrinol 743503, 2015.

4. Zhuo C, Jiang R, Lin X and Shao M: LncRNA H19 inhibits autophagy by epigenetically silencing of DIRAS3 in diabetic cardiomyopathy. Oncotarget 8: 1429-1437, 2017.

5. Holscher ME, Bode C and Bugger H: Diabetic Cardiomyopathy: Does the type of diabetes matter? Int J Mol Sci 17: 2136, 2016.

6. Xu G, Kuang G, Jiang W, Jiang R and Jiang D: Polydatin promotes apoptosis through upregulation the ratio of $\mathrm{Bax} / \mathrm{Bcl}-2$ and inhibits proliferation by attenuating the beta-catenin signaling in human osteosarcoma cells. Am J Transl Res 8: 922-931, 2016.

7. Li Z, Zhang J, Mulholland M and Zhang W: mTOR activation protects liver from ischemia/reperfusion-induced injury through NF-kappaB pathway. FASEB J 31: 3016-3026, 2017.

8. Korkmaz-Icöz S, Al Said S, Radovits T, Li S, Brune M, Hegedús P Atmanli A, Ruppert M, Brlecic P, Lehmann LH, et al: Oral treatment with a zinc complex of acetylsalicylic acid prevents diabetic cardiomyopathy in a rat model of type-2 diabetes: activation of the Akt pathway. Cardiovasc Diabetol 15: 75, 2016.

9. Zhang H, Xiong Z, Wang J, Zhang S, Lei L, Yang L and Zhang Z: Glucagon-like peptide-1 protects cardiomyocytes from advanced oxidation protein product-induced apoptosis via the PI3K/Akt/Bad signaling pathway. Mol Med Rep 13: 1593-1601, 2016.

10. He H, Qiao X and Wu S: Carbamylated erythropoietin attenuates cardiomyopathy via PI3K/Akt activation in rats with diabetic cardiomyopathy. Exp Ther Med 6: 567-573, 2013.

11. Thomas CM, Yong QC, Rosa RM, Seqqat R, Gopal S, Casarini DE, Jones WK, Gupta S, Baker KM and Kumar R: Cardiac-specific suppression of NF-kappaB signaling prevents diabetic cardiomyopathy via inhibition of the renin-angiotensin system. Am J Physiol Heart Circ Physiol 307: H1036-1045, 2014.

12. Wang ZQ, Chen MT, Zhang R, Zhang Y, Li W and Li YG: Docosahexaenoic acid attenuates doxorubicin-induced cytotoxicity and inflammation by suppressing NF-kappaB/iNOS/NO signaling pathway activation in $\mathrm{H} 9 \mathrm{C} 2$ cardiac cells. J Cardiovasc Pharmacol 67: 283-289, 2016.

13. Ren XM, Zuo GF, Wu W, Luo J, Ye P, Chen SL and Hu ZY: Atorvastatin alleviates experimental diabetic cardiomyopathy by regulating the GSK-3beta-PP2Ac-NF-kappaB signaling axis PLoS One 11: e0166740, 2016.

14. Yin C, Deng Y, Gao J, Li X, Liu Y and Gong Q: Icariside II, a novel phosphodiesterase-5 inhibitor, attenuates streptozotocin-induced cognitive deficits in rats. Neuroscience 328: 69-79, 2016.

15. Tian W, Lei H, Guan R, Xu Y, Li H, Wang L, Yang B, Gao Z and Xin Z: Icariside II ameliorates diabetic nephropathy in streptozotocin-induced diabetic rats. Drug Des Devel Ther 9: $5147-5157,2015$
16. Frustaci A, Ciccosanti F, Chimenti C, Nardacci R, Corazzari M, Verardo R, Ippolito G, Petrosillo N, Fimia GM and Piacentini M: Histological and proteomic profile of diabetic versus non-diabetic dilated cardiomyopathy. Int J Cardiol 203: 282-289, 2016.

17. $\mathrm{Zhu} \mathrm{Z}$ and $\mathrm{Hu} \mathrm{X}$ : HMGB1 induced endothelial permeability promotes myocardial fibrosis in diabetic cardiomyopathy. Int J Cardiol 227: 875, 2017.

18. Lebeche D: Diabetic cardiomyopathy: is resistin a culprit? Cardiovasc Diagn Ther 5: 387-393, 2015.

19. Wu H, Sheng ZQ, Xie J, Li R, Chen L, Li GN, Wang L and Xu B: Reduced HMGB 1-mediated pathway and oxidative stress in resveratrol-treated diabetic mice: A possible mechanism of cardioprotection of resveratrol in diabetes mellitus. Oxid Med Cell Longev 2016: 9836860, 2016.

20. Kuo WW, Wang WJ, Tsai CY, Way CL, Hsu HH and Chen LM: Diallyl trisufide (DATS) suppresses high glucose-induced cardiomyocyte apoptosis by inhibiting JNK/NFkappaB signaling via attenuating ROS generation. Int J Cardiol 168: 270-280, 2013.

21. Varga ZV, Giricz Z, Liaudet L, Hasko G, Ferdinandy P and Pacher P: Interplay of oxidative, nitrosative/nitrative stress, inflammation, cell death and autophagy in diabetic cardiomyopathy. Biochim Biophys Acta 1852: 232-242, 2015.

22. Suzuki H, Kayama Y, Sakamoto M, Iuchi H, Shimizu I, Yoshino T, Katoh D, Nagoshi T, Tojo K, Minamino T, et al: Arachidonate 12/15-lipoxygenase-induced inflammation and oxidative stress are involved in the development of diabetic cardiomyopathy. Diabetes 64: 618-630, 2015

23. Zhao YZ, Zhang M, Wong HL, Tian XQ, Zheng L, Yu XC Tian FR, Mao KL, Fan ZL, Chen PP, et al: Prevent diabetic cardiomyopathy in diabetic rats by combined therapy of aFGF-loaded nanoparticles and ultrasound-targeted microbubble destruction technique. J Control Release 223: 11-21, 2016.

24. Yu W, Zha W, Guo S, Cheng H, Wu J and Liu C: Flos Puerariae extract prevents myocardial apoptosis via attenuation oxidative stress in streptozotocin-induced diabetic mice. PLoS One 9: e98044, 2014.

25. Zhang L, Ding WY, Wang ZH, Tang MX, Wang F, Li Y, Zhong M, Zhang Y and Wei Zhang: Erratum to: Early administration of trimetazidine attenuates diabetic cardiomyopathy in rats by alleviating fibrosis, reducing apoptosis and enhancing autophagy. $\mathrm{J}$ Transl Med 14: 309, 2016.

26. Wu MP, Zhang YS, Zhou QM, Xiong J, Dong YR and Yan C: Higenamine protects ischemia/reperfusion induced cardiac injury and myocyte apoptosis through activation of beta2-AR/PI3K/AKT signaling pathway. Pharmacol Res 104: 115-123, 2016.

27. Wang J, Ji SY, Liu SZ, Jing R and Lou WJ: Cardioprotective effect of breviscapine: Inhibition of apoptosis in H9c2 cardiomyocytes via the PI3K/Akt/eNOS pathway following simulated ischemia/reperfusion injury. Pharmazie 70: 593-597, 2015.

28. Liu W, Mao L, Ji F, Chen F, Wang S and Xie Y: Icariside II activates EGFR-Akt-Nrf2 signaling and protects osteoblasts from dexamethasone. Oncotarget 8: 2594-2603, 2017.

29. Jiang C, Tong YL, Zhang D, Liu LZ and Wang JF: Sinomenine prevents the development of cardiomyopathy in diabetic rats by inhibiting inflammatory responses and blocking activation of NF-kappaB. Gen Physiol Biophys 36: 65-74, 2017.

30. Song J, Feng L, Zhong R, Xia Z, Zhang L, Cui L, Yan H, Jia X and Zhang Z: Icariside II inhibits the EMT of NSCLC cells in inflammatory microenvironment via down-regulation of Akt/NF-kappaB signaling pathway. Mol Carcinog 56: 36-48, 2017.

31. Lee KS, Lee HJ, Ahn KS, Kim SH, Nam D, Kim DK, Choi DY, Ahn KS, Lu J and Kim SH: Cyclooxygenase-2/prostaglandin E2 pathway mediates icariside II induced apoptosis in human PC-3 prostate cancer cells. Cancer Lett 280: 93-100, 2009. 